\section{REPRESENTAÇÕES SOCIAIS DO CUIDADO À PESSOA 'VIVENDO' COM HIV/AIDS: ESTRUTURA E CO-OCORRÊNCIA}

\section{SOCIAL REPRESENTATIONS OF THE CARE OF THE PERSON 'LIVING' WITH HIV/AIDS: STRUCTURE AND CO-OCCURRENCE}

Cleuma Sueli Santos Suto ${ }^{1, *} /$ Carle Porcino ${ }^{2}$ / Mirian Santos Paiva ${ }^{3}$ / Washington da Silva Santos ${ }^{4} /$ Gizélia dos Santos Souza ${ }^{5}$

\section{INTRODUÇÃO}

$\mathrm{O}$ cuidado pode ser entendido como o modo-de-ser essencial da humanidade. Está presente em tudo e foi discutido no século passado como um ideal filosófico.Atualmente, pode ser compreendido a partir de conceitos amplos e diversos como a atenção, o zelo e o ato de assistir; sendo mais do que um ato é uma atitude de responsabilidade e envolvimento afetivo (CHERNICHARO, 2013).

No entanto, as necessidades de saúde expressam as carências de milhões de indivíduos e também, simultaneamente, as necessidades coletivas de cuidado. $\mathrm{O}$ processo de determinação das carências individuais pode ser vivenciado com o grupo, desse modo, alcança uma dimensão coletiva. Trata-se, pois, de uma mediação fundamental da práxis social inerente à vida em sociedade (COLLIÈRE, 2003).

${ }^{1}$ Doutoranda no Programa de Pós-Graduação em Enfermagem e Saúde, Escola de Enfermagem, Universidade Federal da Bahia, Salvador, Bahia; Departamento de Educação, Campus VII, Universidade do Estado da Bahia, Senhor do Bonfim, Bahia - Brasil

${ }^{2}$ Doutoranda no Programa de Pós-Graduação em Enfermagem e Saúde, Escola de Enfermagem, Universidade Federal da Bahia, Salvador, Bahia; Associação de Travestis de Salvador/Grupo Gay da Bahia, Salvador, Bahia - Brasil.

${ }^{3}$ Docente, Programa de Pós-Graduação em Enfermagem e Saúde, Escola de Enfermagem, Universidade Federal da Bahia, Salvador, Bahia - Brasil

${ }^{4}$ Docente, Departamento de Saúde, Universidade Estadual do Sudoeste da Bahia, Jequié, Bahia - Brasil.

${ }^{5}$ Graduanda em Enfermagem, Campus VII, Universidade do Estado da Bahia, Senhor do Bonfim, Bahia - Brasil

*E-mail para correspondência: cleuma.suto@gmail.com

\section{RESUMO}

O cuidado a pessoas vivendo com o vírus da imunodeficiência adquirida vem se modificando ao longo dos anos diante de descobertas cientificas. Objetivou-se analisar o conteúdo e a estrutura das representações sociais de profissionais de saúde acerca do cuidado a pessoas vivendo com HIV/AIDS. Pesquisa qualitativa fundamentada na Teoria das Representações Sociais. Coletaram-se evocações livres de 73 profissionais de saúde de serviços públicos especializados, em Salvador-Bahia, submetendo-as a Análise de co-ocorrência por meio do software EVOC. Os termos 'acolhimento', 'aconselhamento', 'capacitação' e 'educação-saúde' foram sinalizados como estruturantes do núcleo central. Apreendeu-se no universo semântico o cuidado como atitudes positivas e incorporadas ao discurso institucional. Conclui-se que para o grupo estudado o cuidado a pessoa que vive com HIV/AIDS é evidenciado em seu conteúdo e estrutura pela proposta normatizada nas políticas de humanização e enfrentamento da AIDS.

Palavras-chave: Cuidado; Profissionais de saúde; Representações sociais; HIV; Síndrome da Imunodeficiência adquirida.

\section{ABSTRACT}

The care of people living with human immunodeficiency virus has been changing over the years before scientific discoveries. This study aimed to analyze the content and structure of social representations of health professionals about the care of people living with HIV/AIDS. Qualitative research based on the Theory of Social Representations. Collected up free evocations of 73 health professionals specialized public services in Salvador, Bahia, subjecting them to co-occurrence Analysis by EVOC software. The terms 'reception, counseling, training and education-health' have been flagged as structuring of the central core. He was seized in the semantic universe care as positive attitudes and incorporated into the institutional discourse. It is concluded that the group studied the care the person living with HIV/AIDS is evident in its content and structure proposed by the humanization of standardized policies and responding to AIDS.

Keywords: Care; Health professionals; Social representations; HIV; Acquired immunodeficiency syndrome

Submetido em: 04 de jun. 2018

Aceito em: 06 de nov. 2018 
com Ciência

Uma revista multidisciplinar
Para a antropologia da doença, o corpo é um objeto de estudo, e tanto o corpo, quanto a doença, passa a ser objeto de conhecimento científico. Por esse ângulo, a razão científica propõe vencer o corpo doente e dominar e/ou eliminar a doença. Tal concepção, ao encontrar amparo nas instituições assistenciais, imputa uma inversão de valores, a tal ponto que as tecnologias de investigação e de tratamentos passaram a ser objeto das práticas de cuidado ligado ao ser humano. Nessa acepção, a doença deve ser detida e erradicada, para isso, se faz necessária a medicalização da vida, alvitrada pelo tratamento (BIEHL, 2011). No que concerne as questões relacionadas à AIDS, os fenômenos de medicalização e mercantilização, que têm uma importância reconhecida, se interpõem ao cuidado.

Avanços científicos e tecnológicos proporcionaram controle e tratamento da doença, possibilitando melhoria da qualidade de vida das pessoas infectadas pelo vírus da imunodeficiência humana (HIV) e com a síndrome da imunodeficiência adquirida (AIDS). Classificada atualmente como uma doença crônica pelas limitações físicas e psicológicas, continua provocando impactos diretos onde o estigma se mostra associado a essas vivências (SILVEIRA; CARVALHO, 2011). De modo que os profissionais de saúde necessitam apresentar respostas a questões até então pouco exploradas no cuidado à saúde, como as sexualidades, perdas, morte e outras condutas socialmente censuráveis, impondo a obrigação de reformular a estrutura do cuidado em saúde (SOUZA; SILVA, 2013).

A epidemia do HIV/AIDS nos seus primórdios era tratada como uma questão essencialmente biomédi- ca. Recentemente, compreende-se como um evento coletivo e um constructo social, que se molda dentro do contexto de sistemas sociais, culturais, políticos e econômicos, altamente específicos, interligados e sobrepostos (OLIVEIRA, 2011).

Para melhor compreender tais questões, consideramos que a produção no campo das ciências sociais e da saúde aplicada ao estudo das representações sociais sobre a AIDS, é reconhecida como importante no cenário acadêmico brasileiro. No entanto, em busca realizada na base de dados $\mathrm{Sci}$ entific Electronic Library Online (SciELO), em 2016, a análise do cuidado desenvolvido com os indivíduos vivendo com HIV/AIDS não recebeu a mesma preocupação no cenário nacional. Observou-se uma produção científica ainda pouco expressiva e inespecífica, apontando um possível 'desconhecimento' sobre as práticas profissionais associadas a AIDS e as possíveis transformações do cuidado ao longo da epidemia, no que concerne as subjetividades que perpassam o fenômeno.

Os conteúdos das representações sociais dos profissionais de saúde sobre o cuidado a pessoas com HIV/AIDS devem permear questões sociais (diálogo, relação profissional/usuário) e questões gerenciais (infraestrutura, recursos humanos). De modo, que as relações interpessoais despontam como possível indicador de qualidade de humanização do cuidado.

Nessa acepção, concordamos com Minayo (2013, p.91) ao afirmar que as representações podem ser olhadas como "matéria-prima para análise do social e também para ação pedagógico-política de transformação, pois retratam e refratam a realidade segundo determinados segmentos da sociedade". Aspectos esses, que possibilitam a realização de estudos em torno de questões relacionadas à saúde, considerando a perspectiva psicossocial, a partir da Teoria das Representações Sociais (TRS) (MOSCOVIVI, 2012).

Assim, coloca-se como questão: Como profissionais de saúde representam o cuidado a pessoas vivendo com HIV/AIDS? Acreditando que o conhecimento de elementos que compõem as representações sociais podem contribuir no reconhecimento de nexos entre as práticas de cuidado e o contexto cultural vivenciado pelo(a)s profissionais de saúde acerca da problemática da AIDS e, consequentemente, sobre as práticas do cuidado, delimitou-se como objeto de estudo: representações sociais de profissionais de saúde acerca do cuidado à pessoas 'vivendo' com HIV/AIDS.

Os dados ora apresentados, constituem recorte de uma pesquisa mais ampla, financiada pelo Conselho Nacional de Desenvolvimento Científico e Tecnológico ( $\mathrm{CNPq})$. $\mathrm{O}$ artigo tem como objetivo analisaro conteúdo e a estrutura das representações sociais de profissionais de saúde acerca do cuidado a pessoas vivendo com HIV/AIDS.

Este estudo torna-se relevante para a Enfermagem por facultar reflexões em torno da práxis e minimizar as lacunas existentes entre a teoria e a prática profissional. Nesse seguimento, poderá apoiar as práticas de profissionais que atuam em unidades de saúde, com atendimento a pessoas vivendo com HIV/AIDS, especialmente, no que se relaciona, aos impactos de tal representação sobre as práticas de cuidado.

\section{METODOLOGIA}

Estudo descritivo e analítico, com abordagem qualitativa, fundamentado na TRS como um sistema de referência que permite a interpretação dos 
acontecimentos reais, dando sentido à vivência profissional, sendo uma ferramenta indispensável à identificação dos aspectos cognitivos, afetivos, sociais e simbólicos do conhecimento do senso comum, considerando a comunicação e interação sociais (MINAYO, 2014). Este estudo é parte dos resultados da dissertação de mestrado intitulada "Representações sociais de profissionais de saúde sobre as práticas do cuidado em HIV/AIDS" defendida em 2015, junto ao Programa de PósGraduação em Enfermagem e Saúde (PPGENF) da Universidade Federal da Bahia (UFBA), em parceria com a Universidade do Estado do Rio de Janeiro (UERJ).

Definida como um conjunto de conceitos articulados, a TRS, tem origem nas práticas sociais e diversidades grupais cujas funções são dar sentido à realidade social, produzir identidades, organizar as comunicações e orientar as condutas (SANTOS; ALMEIDA, 2005). A TRS mostra o sujeito, de modo indissociável, construindo o seu mundo na tentativa de adaptação às modificações da realidade social, influenciado pela cultura, ideologia e valores, que permitem ser, os indivíduos, simultaneamente, produto e produtor da sociedade, em constante relação com o outro (MOSCOVICI, 2013).

Neste artigo, toma-se como aporte teórico a abordagem estrutural da TRS. Tal abordagem, parte do pressuposto que os elementos da representação social com importância em sua estrutura são mais prototípicos, isto é, mais acessíveis à consciência (WACHELKE; WOLTER, 2011). Como proposta para abordagem estrutural da TRS, trabalharemos com o Núcleo Central (NC), conforme descrito por Abric (2005). O NC é o elemento essencial de toda representação, pode encontrar sua origem diretamente nos valores que transcendem e que não exigem nem aspectos figurativos, nem esquematizações, nem mesmo concretização.

Para a coleta de dados, utilizou-se um instrumento composto de duas partes: a primeira, com os dados sociodemográficos que caracterizaram os participantes da pesquisa e a segunda, o Teste de Associação Livre de Palavras (TALP), a partir do estímulo indutor: cuidado a pessoa com HIV/AIDS, por considerá-lo suficiente para o alcance do objetivo proposto.

O TALP é caracterizado como uma técnica projetiva e visa identificar as dimensões latentes das representações sociais por meio dos elementos constituintes da rede associativa dos conteúdos elaborados e relacionados ao estímulo indutor (NÓBREGA; COUTINHO, 2011). Além de permitir que as expressões do sujeito não sejam compreendidas apenas a partir do significado que o próprio sujeito conscientemente lhe confere, e sim interpretadas em termos de alguma conceituação psicológica pré-estabelecida (OLIVEIRA et al., 2005).

Participaram deste estudo profissionais de saúde de nível superior, de quatro unidades da rede pública de Serviços de Assistência Especializada (SAE) na cidade do Salvador-Bahia, escolhidos por serem referência na atenção e/ou cuidado à pessoa vivendo com HIV/AIDS. Os critérios de inclusão foram ter vínculo permanente com a instituição; estar em efetivo exercício no período de coleta de dados; ter, pelo menos, um ano de trabalho na instituição, possibilitando assim, a elaboração das RS.

Os dados foram coletados no período de dezembro 2014 a março 2015. Utilizamos as variáveis: sexo, idade, profissão, tempo de formação e tempo de atuação profissional, para caracterização das participantes. Para o processamento dos dados evocados utilizou-se o software Ensemble de Programmes Permettantl'Analyse de Évocations - EVOC, versão 2005. Esta ferramenta computacional potencializa a validade do estudo ao permitir a possibilidade de procedimentos de transferências dos resultados para outros estudos, como por exemplo, no caso das análises: lexical e similaridades. Possibilita também, a realização dos cálculos das médias e coocorrência e construção do quadro de quatro casas, por meio do qual é possível caracterizar o núcleo central e os elementos periféricos da representação (SARUBBI JUNIOR et al., 2013).

No primeiro momento, o tratamento de equivalência dado às respostas evocadas foi agrupá-las por critérios semânticos, de modo que, foram classificadas conforme um significado em comum. Seguindo-se a análise pelo EVOC, foi calculada a frequência e ordem de aparição dos termos produzidos, na representação dos profissionais de saúde, por fim elaborou-se a categorização e matriz de co-ocorrência.

Ainda com vistas à melhor caracterização da estrutura das representações sociais dos profissionais participantes do estudo, nos valemos de outros recursos do software EVOC para determinação de co-ocorrência. Essa técnica foi desenvolvida para caracterizar a estrutura de uma representação social obtida a partir de evocações de palavras que se constitui de duas etapas; a primeira, chamada análise prototípica, baseia-se no cálculo de frequências e ordens de evocação das palavras, enquanto que uma segunda etapa centra-se na formulação de categorias englobando as evocações que avalia suas frequências, composições e 
com Ciência

Uma revista multidisciplinar co-ocorrência, que são acessadas a partir do programa CATEVOC/REDOCAT/CATINI do software EVOC 2005 (VERGÈS, 1992; WACHELKE; WOLTER, 2011).

A análise de co-ocorrência possibilita caracterizar a estrutura da representação das evocações ao indicar a 'força' de ligação entre os aglomerados e de sua importância relativa ('centralidade' e 'densidade') (CALDEIRA, 2007). De modo complementar, na análise das evocações, pode ser útil registrar a proporção de ocorrências de cada forma na ordem de evocação, como demonstrado neste estudo em quadro elaborado pelas autoras.

Para fins de atendimento aos princípios éticos na pesquisa houve aprovação pelo Comitê de Ética e Pesquisa (CEP) da Escola de Enfermagem da UFBA, sob $n^{\circ} 874.468$ e CAAE: 37189014.8 .0000 .5531 .

\section{RESULTADOS E DISCUSSÃO}

Compreender a subjetividade de trabalhadoras/es da saúde em uma perspectiva psicossocial exigiu inicialmente descrever os contextos em que exercem suas atividades. $\mathrm{O}$ banco de dados foi composto por variáveis fixas e categorização das evocações de 73 participantes de quatro organizações. A amostra foi formada por 23 enfermeiras $(31,5 \%), \quad 15$ médica(o)s $(20,5 \%)$ e 35 profissionais classificados como outros (assistente social, nutricionista, odontóloga(o), psicólo$\mathrm{ga}(\mathrm{o})$, farmacêutica(o), fisioterapeuta e terapeutas ocupacionais) perfazendo $48 \%$ do total das/dos participantes.

Foram evocadas 342 palavras pelas/os 73 participantes, das quais 102 diferentes, após o agrupamento de acordo com o significado semântico. Sendo processadas pelo software para elaboração do quadro de quatro casas e matriz de co-ocorrênciapor meio do programa CATINI. O tratamento dos dados foi efetuado tomando como referência a frequência mínima de $4 \mathrm{e}$ a acumulada de $64,6 \%$ e como Ordem Média de Evocação (OME) 2,9, permitindo assim, apreender as RS do grupo investigado.

O cruzamento entre frequência e hierarquização das evocações, segundo Oliveira et al., (2005), possibilita a formação de um quadro de quatro casas, que expressa o conteúdo e a estrutura das representações sociais. A interpretação dos dados, com base no quadro e na matriz de co-ocorrência será apresentada, no intuito de ampliar a validação dos achados, pautada nos pressupostos teóricos da abordagem estrutural das RS.

A análise do corpus foi formada pelas evocações de todas/os as/os participantes da pesquisa em resposta direta ao termo indutor "Cuidar de pessoa com HIV/AIDS". Foram desprezadas as evocações cuja frequência foi igual ou inferior a 2 , encontrou-se a frequência média de evocação igual a 9. A análise combinada desses dados resultou no quadro de quatro casas apresentado na Figura 1.

De acordo com Abric (2005), o quadrante superior esquerdo é constituído por palavras que, muito provavelmente, tenham uma maior importância no esquema cognitivo dos sujeitos, sendo consideradas como o núcleo central da representação. Este quadrante está composto por palavras com alta frequência e baixa ordem de evocação. Colocada como o mais importante componente de acordo com sua hierarquia, os termos 'aconselhamento' e 'acolhimento' constituem o possível núcleo central da representação e, se confirmam também por suas saliências. Sendo acompanhados pelos termos 'capacitação' e 'educação-saúde' que conferem homogeneidade ao núcleo central.

O conhecimento compartilhado por essas/es profissionais caracteriza-se por conceber o cuidar como uma atividade que necessariamente passa pelo envolvimento com a pessoa que vive com HIV/AIDS, para tanto exige ações de capacitação e educação. E coaduna com Chernicharoet al., (2013) ao afirmarem que a Política Nacional de Humanização (PHN) apresenta em seu bojo o conceito de ambiência que se refere ao tratamento oferecido no espaço físico, entendido como espaço social, profissional e de relações interpessoais, o qual deve proporcionar ação acolhedora, resolutiva e humana.

Para Formozo e Oliveira (2010) o núcleo central é resultado da memória coletiva e do sistema de normas ao qual certo grupo faz alusão, constituindo-se em prescrições absolutas. Esta representação reproduz a associação do cuidado com as políticas públicas de controle da epidemia da AIDS e de humanização, ligadas diretamente à possibilidade de convívio com a AIDS enquanto uma doença crônica. Expressando dessa forma, o caráter normativo da representação dessas/es profissionais de saúde.

Aqui, o aconselhamento é entendido como um processo de escuta ativa, individualizado e centrado no cliente, com vista a uma relação de confiança entre o profissional e a pessoa vivendo com AIDS (ABRÃO et al., 2014). Tanto aconselhamento quanto acolhimento advêm do discurso 
Figura 1: Quadro de Quatro Casas ao termo indutor Cuidar de pessoa vivendo com HIV/AIDS em ordem direta no conjunto dos participantes estudados. Salvador, 2018.

\begin{tabular}{|l|l|c|c|l|c|c|}
\hline \multirow{2}{*}{$\begin{array}{l}\text { Fre- } \\
\text { quência } \\
\text { Média }\end{array}$} & Termo evocado & Freq. & OME & Termo evocado & Freq. & OME \\
\cline { 2 - 7 }$\geq 9$ & Acolhimento & 20 & 2,600 & Ajuda & 13 & 3,615 \\
& Aconselhamento & 24 & 2,875 & Amor & 12 & 2,917 \\
& Capacitação & 16 & 2,750 & Compromisso & 9 & 3,222 \\
& Educação-saúde & 14 & 2,714 & Cuidado & 11 & 3,455 \\
\hline$<9$ & Atendimento- & 8 & 2,250 & Aceitação & 7 & 3,286 \\
& humanizado & & & Adesão-tratamento & 4 & 3,000 \\
& Atenção & 7 & 2,000 & Apoio-psicológico & 4 & 3,250 \\
& Cuidado-proteção & 6 & 2,667 & Empatia & 5 & 3,400 \\
& Disponibilidade & 4 & 2,750 & Não-discriminação & 4 & 3,500 \\
& Paciência & 8 & 2,250 & Prevenção & 6 & 3,167 \\
& Reconhecimento & 7 & 2,571 & Solidariedade & 7 & 3,286 \\
& Respeito & 7 & 2,143 & Tratamento & 6 & 3,000 \\
& & & & &
\end{tabular}

difundido a partir da PHN e, podem ser propagados. Pois, de acordo com Moscovici (2012) as pessoas adquirem um repertório comum de interpretações, regras e procedimentos que podem ser empregadas à vida cotidiana, de forma semelhante, as expressões linguísticas são livres e possíveis a todos.

Nesse sentido, Moscovici (2012, p. 208) reitera que "[...] as representações sociais têm como finalidade primeira e fundamental tornar a comunicação, dentro de um grupo relativamente não problemático e reduzir o "vago" considerando certo grau de consenso entre seus membros". No entanto, para se ter uma assistência humanizada não satisfaz investir em equipamentos e tecnologias, deve-se investir também no acolhimento, baseado na comunicação/diálogo e respeito, como proposto pela $\mathrm{PNH}$.

Outro aspecto que vale ser salientado é o predomínio de questões afetivas que perpassam o as ações esperadas no acolhimento, nesta representação. Os termos evidenciados no núcleo central, apontam "instrumentos” (capacitação e educação-saúde) como possibilidades de enfrentamento do fenômeno da AIDS.

O conceito de cuidado é polissêmico e atravessado pelas concepções de saúde e doença, exemplificando, no campo da saúde, ele indica um objeto complexo, sintético, emergente, multifacetado além de extravasar os recortes disciplinares de ciência (GOMES et al., 2011). Considerando que os profissionais têm a função de prestar assistência a pessoas sadias ou doentes, famílias e comunidades, no desempenho de atividades para promoção, manutenção e recuperação da saúde a revelação desse sentido pode interferir positivamente na adoção de condutas clínicas e/ou práticas de cuidado por parte dos profissionais as/aos usuárias/os dos serviços de saúde especializados.

A partir dos elementos constituintes da representação pode-se afirmar que os profissionais corroboram uma visão do cuidado a pessoa vivendo com HIV/AIDS possivelmente estabelecida após a introdução da terapia antirretroviral. Para Cohen e Gay (2010) considerar a AIDS uma doença crônica significa que seu manejo e tratamento adequado, diminui a proba- bilidade de adoecimento e morte, prolongando a convivência com o agravo.

As demais palavras que aparecem no segundo quadrante ou primeira periferia (ajuda, amor, compromisso e cuidado) despontam um posicionamento, também positivo frente à problemática da AIDS e o processo de cuidar. Os termos denotam aproximação e empatia diante do agravo, pelas pessoas abrangidas no interior do fenômeno e coaduna com o contexto atual da AIDS. Tais elementos revelam o fortalecimento de ações interdisciplinares com reflexos no campo dos direitos humanos para mudanças nas práticas de cuidado.

No entanto, como as representações não estão nunca limitadas a uma simples circunscrição de seus conteúdos, como explicitado por Moscovici (2012), se faz necessário estabelecer a conjuntura em que são concebidas. Este autor também salienta que nossas representações são sempre filtradas por meio do discurso de outros, das experiências que vivemos e da coletividade as quais pertencemos.

Estudiosos da TRS chamam atenção para a possibilidade de alguns elementos centrais aparecerem, no quadro de quatro casas, como componentes da primeira periferia, por serem termos com saliência (SÁet al.,2009), como parece ser o caso do elemento amor $(2,917)$ nesta representação.

Os termos constituintes da zona de contraste e da periferia mais distante (atendimento-humanizado, paciência, respeito, aceitação e solidariedade) possuem frequência baixa e alta OME, sendo evocados por um pequeno número de participantes, no entanto, robustecem o núcleo central em seu aspecto normativo/afetivo. A dimensão psicossocial que emerge desses elementos, apontam na direção da criação e fortalecimento de víncu- 
com Ciência

Uma revista multidisciplinar los, de modo a estabelecer uma relação de empatia que permitirá ao paciente revelar questões de seu adoecimento ou a minimização do nível de ansiedade e a diminuição da carga de estresse.

Ayres (2004) afirma que o cuidado à saúde tem grande potencialidade para subverter o paroxismo objetificador das pessoas nas práticas de saúde em decorrência da intersubjetividade viva no momento assistencial, ou seja, o cuidado permite uma troca efetiva que se estabelece no espaço relacional, que extrapola o espaço tecnológico que permeia a assistência.

A co-ocorrência traz um componente contextual para a análise uma vez que os pares são formados a partir da presença das palavras em uma mesma linha, evocadas pelo mesmo participante. Trata-se, também, de um recurso auxiliar, oferecido pelo EVOC, para a inferência do tema no corpus analisado. No Quadro 1, pode-se visualizar a 'força' de associação entre os pares de termos: 'acolhimento e cuidado', representada pela quantidade de evocações do termo 'acolhimento' (20) e o número de vezes que aparece junto as palavras 'aconselhamento' (8x) e ‘educação' (6x). Por sua vez, o termo 'cuidado' apresentou 11 evocações, mantendo-se próxima de 'acolhimento e aconselhamento'(4x) e 'amor e educação’ (3x).
Os termos 'amor e tratamento' ‘ajuda e aconselhamento' e, 'cuidado e ajuda' tiveram uma co-ocorrência muito fraca, uma vez que o valor do coeficiente de associação foi inferior a 0.01 , mostrando que esses pares apresentam uma afinidade muito baixa. Sendo a correlação uma medida da dependência linear dos termos evocados por um participante, seus altos valores implicam uma relação linearentre os termos, o que não ocorreu, como no exemplo acima com 'amor e tratamento'.

A análise de co-ocorrências entre pares de palavras permite estabelecer índices estatísticos que representam a força de associação entre esses pares e, a partir dos valores encontrados mapear o estado de uma área do conhecimento num determinado momento além da elaboração de diversos tipos de representações gráficas (CALDEIRA, 2007).Para o estímulo "cuidar de pessoa com HIV/AIDS" foi gerada a seguinte organização a partir da análise da matriz de co-ocorrência, Figura 2.

$\mathrm{Na}$ matriz de preferência, também elaborada pelo programa CATINI, foi possível verificar que termo 'acolhimento' aparece 4x na frente a 'aconselhamento' e, 'educação-saúde' $3 x$ antes de 'acolhimento' que por sua vez aparecer $2 \mathrm{x}$ depois de 'cuidado'.
Os elementos estatísticos confirmam a centralidade do termo 'acolhimento', através do cálculo da matriz de preferência. Neste estudo, é possível afirmar que o termo 'acolhimento' ocupou a $1^{\mathrm{a}}$ posição em $40 \%$ das vezes que foi evocado, demonstrando estar incorporado ao inconsciente dos profissionais. Fato semelhante ocorreu com o termo 'educação-saúde' o mesmo ocupou a $1^{\mathrm{a}}$ posição em $36 \%$ das vezes em que foi evocado, sugerindo estarem ancorados nas bases legais que regulamentam a política de humanização da assistência.

Vale ressaltar que o acolhimento institui um desafio na construção de um cuidado integral e é elemento de fundamental valor para a qualidade do serviço. Para Araújo et al., (2011) deve ser apreciado como ferramenta essencial para o estabelecimento de um processo de trabalho diferenciado e para a solidificação de relações humanitárias entre usuários e profissionais.

Em relação ao cuidado às pessoas vivendo com HIV/AIDS os especialistas reconhecem a necessidade - e a urgência - de ampliar o acesso a preservativos, a testes e a serviços de saúde e intensificar as ações de aconselhamento sobre as formas de prevenir a infecção e ampliar as estratégias de prevenção. Neste estudo encontra-

Quadro 1: Matriz de Co-ocorrência do programa CATINI, fornecida pelo software EVOC 2005, para o termo indutor Cuidar de pessoas vivendo com HIV/AIDS, Salvador, 2018 (Adaptada pela autora).

\begin{tabular}{|c|c|c|c|c|c|c|c|c|}
\hline Categoria & acolhimento & capacitação & amor & educação & aconselhamento & ajuda & tratamento & cuidado \\
\hline Acolhimento & 0 & 5 & 3 & 6 & 8 & 3 & 5 & 4 \\
\hline Capacitação & 5 & 0 & 2 & 2 & 3 & 2 & 2 & 2 \\
\hline Amor & 3 & 2 & 0 & 2 & 3 & 1 & $\mathbf{0}$ & 3 \\
\hline Educação & 6 & 2 & 2 & 0 & 5 & 3 & 2 & 3 \\
\hline Aconselhamento & 8 & 3 & 3 & 5 & 0 & 5 & 2 & 4 \\
\hline Ajuda & 3 & 2 & 1 & 4 & 0 & 0 & 2 & $\mathbf{0}$ \\
\hline Tratamento & 5 & 2 & $\mathbf{0}$ & 2 & 2 & 2 & 0 & 1 \\
\hline Cuidado & 4 & 2 & 3 & 3 & 4 & 0 & 1 & 0 \\
\hline
\end{tabular}

Rev. ComCiência - dez. 2018, vol. 3, no. 3, p. 23-31 / doi: 10.36112/issn2595-1890.v3i1.p23-31 
Figura 2: Quadro de co-ocorrência a partir do programa CATINI do software EVOC 2005 para o termo indutor Cuidar de pessoas vivendo com HIV/AIDS, Salvador, 2018 (Adaptada pela autora)

Amor

\section{Cuidado}

Educação-saúde

\section{Acolhimento}

\section{Aconselhamento}

Tratamento

mos no universo semântico dos profissionais palavras que traduzem o cuidado como resposta ao esperado pela sociedade, por especialistas, pelas políticas de saúde e pelo(a)s pessoas que vivem com HIV/AIDS.

Os resultados evidenciam também que a representação de cuidar de pessoa com HIV/AIDS guarda estreita relação com a promoção da vida e a humanização dos cuidados em saúde. A esse respeito, Suto et al., (2017) pontuam que a efetivação do processo de humanização suscita "[...] meios que culminem na transformação do processo e das condições de trabalho de profissionais de saúde". Assim, à medida que se remete à complexidade e integralidade no processo de assistir em serviços de saúde, Borges e Silva (2010), apontam sobre a importância para a relação entre acolhimento, aconselhamento e educação-saúde como uma tríade a ser seguida nas práticas cotidianas de cuidado.

\section{CONCLUSÃO}

Frente aos resultados, o cuidado a pessoa que vive com HIV/AIDS é evidenciado em seu conteúdo e estrutura pela proposta normatizada nas políticas de humanização e enfrentamento da AIDS. As mudanças no espaço da formação profissional e a complexidade que permeia o campo de sabres e poderes na área da saúde, possivelmente também tenham contribuído para a construção da representação de um cuidar qualificado e sensível, objetivado em 'acolhimento e aconselhamento'. Ratificado na análise de co-ocorrência ao evidenciar a centralidade do termo 'acolhimento' e sua densidade junto à semântica 'cuidado' .

As/Os participantes referemse ao cuidado através de representações que lhe conferem caráter normativo, prescrevendo atitudes e comportamentos socialmente construídos e aceitos como próprios de profissionais de saúde, enfatizando a construção histórica em oposição à sociedade em geral. Estes resultados indicam a necessidade de estratégias que contribuam no aprimoramento de políticas direcionadas ao enfrentamento deste agravo.

O presente artigo apresenta elementos, aportados teoricamente, que indicam a incorporação da política nacional de humanização nas práticas assistenciais, precisamente por compreender que concepção ancoradas nas políticas estão compondo o cotidiano do processo de cuidar de pessoas vivendo com HIV/AIDS. Entretanto é preciso assinalar que tais resultados não podem ser generalizados, haja vista o cenário restrito em que o estudo foi realizado - serviços de atenção especializados, apresentando-se, por conseguinte como uma limitação do estudo.

Assim, é imprescindível o investimento em inquirições futuras acerca do cuidado e o HIV/AIDS, utilizando a TRS ou outras teorias e metodologias, abrangendo outros cenários, como a atenção básica para assim confirmar ou confrontar tais resultados.

\section{FINANCIAMENTO}

A presente pesquisa recebeu financiamento da Fundação de Amparo à Pesquisa do Estado da Bahia (FAPESB).

\section{REFERÊNCIAS}

ABRÃO, F.M.S.et al. Características estruturais e organizacionais de serviços de assistência especializada em HIV/AIDS na cidade de Recife, Brasil.

Revista Baiana de Saúde Pública, v.38, n.1, p.140-154. jan./mar. 2014. Disponível em:

<http://rbsp.sesab.ba.gov.br/index.php/ rbsp/article/view/702/1161>. Acesso em: 20 mar. 2017.

ABRIC, Jean-Claude. A zona muda das representações sociais. In: OLIVEIRA,DenizeCristina;CAMPOS, Pedro Humberto Faria.

(Org.).Representações sociais: uma teoria sem fronteiras. Rio de Janeiro. Museu da República, 2005. P.27-38.

ARAÚJO, M.A.L.;ANDRADE, R.F. V.; MELO, S.P. O acolhimento como estratégia de atenção qualificada: percepção de gestantes com HIV/AIDS em Fortaleza, Ceará. Revista Baiana de Saúde Pública,v. 35, n. 3, p.710721, set. 2011. Disponível em: <http://rbsp.sesab.ba.gov.br/index.php/ 
com Ciêncio

Uma revista multidisciplinar rbsp/article/view/327/294>. Acesso em: 20 mar. 2017.

AYRES, J.R.C.M. Cuidado e reconstrução das práticas de saúde. Interface (Botucatu), v.8, n.14, p.7392,2004.Disponível em: <http://www.scielo.br/pdf/icse/v8n14/ v8n14a04.pdf >. Acesso em: 20 mar. 2017.

BIEHL, J. Antropologia no campo da saúde global. Horiz antropol., Porto Alegre, v. 17, n. 35, p. 227-256, jun. 2011.Disponível em: <http://www.scielo.br/pdf/ha/v17n35/v 17n35a09.pdf $>$. Acesso em: 17 jun. 2017.

BORGES, M. S.; SILVA, H. C. P. Cuidar ou tratar? Busca do campo de competência e identidade profissional da enfermagem. Revbrasenferm., Brasília, v. 63, n. 5, p. 823-829, setout, 2010.Disponível em: < $\underline{\text { http://www.scielo.br/pdf/reben/v63n5 }}$ 121.pdf >. Acesso em: 20 fev. 2017.

\section{CALDEIRA, Silvia MariaG.Lendo} Bohr ao pé da letra: análise de elementos conceituais em escritos de Niels Bohr. Salvador; Feira de Santana, Bahia. 2007. 143f. Dissertação (Mestrado em Ensino, Filosofia e História das Ciências)- Universidade Federal da Bahia, Universidade Estadual de Feira de Santana, Salvador, Feira de Santana.

\section{CHERNICHARO; I. M.;FREITAS,} F.D. S.;FERREIRA, M.A. Humanização no cuidado de enfermagem: contribuição ao debate sobre a Política Nacional de Humanização. Rev. bras. enferm., Brasília, v. 66, n. 4, p. 564570, ago., 2013. Disponível em: <http://www.scielo.br/pdf/reben/v66n4 /v66n4a15.pdf>. Acessoem: 10 jul. 2017.

COHEN, M.S.;GAY, C.L. Treatment to Prevent Transmission of HIV-

1.Oxford Journals. ClinicalInfectiousDiseases.v. 50, supl. 3, p. 85-95, 2010.Disponível em: $<$ https://academic.oup.com/cid/article/ 50/Supplement 3/S85/322265>. Acesso em:11 jul. 2017.

COLLIÈRE, Marie-Françoise. Cuidar...A primeira arte da vida. 2.ed.Loures, Portugal: LUSOCIÊNCIA, 2003.

FORMOZO, G. A.; OLIVEIRA, D. C. Representações sociais do cuidado prestado aos pacientes soropositivos ao HIV. Rev. bras. enferm., Brasília, v. 63, n. 2, abr., 2010. Disponível em: <http://www.scielo.br/pdf/reben/v63n2 /10.pdf $>$. Acesso em: 20 jun. 2017.

GOMES, A. M. T.et al. As facetas do convívio com o HIV: formas de relações sociais e representações sociais da AIDS para pessoas soropositivas hospitalizadas. Esc. Anna Nery, Rio de Janeiro, v. 16, n. 1, p. 111-120, mar. 2012. Disponível em:

<http://www.scielo.br/pdf/ean/v16n1/v 16n1a15.pdf $>$. Acesso em: 20 jun. 2017.

MINAYO, Maria Cecília de Souza. $O$ desafio do conhecimento: pesquisa qualitativa em saúde. 14. ed. São Paulo: Hucitec, 2014.

MOSCOVICI, Serge. Representações sociais:investigações em psicologia social. 9.ed. Petrópolis, Rio de Janeiro, RJ: Vozes, 2012.

NÓBREGA, Sheva Maia da; COUTINHO, Maria da Penha de Lima. A técnica de associação livre de palavras. In: COUTINHO, Maria da Penha de Lima; SARAIVA, Evelyn Rúbia de Albuquerque. (Org.) Métodos de pesquisa em psicologiasocial: perspectivas qualitativas e quantitativas. João Pessoa: Editora Universitária, 2011, p. 95-106.

OLIVEIRA, DenizeCristina de.et al. Análise das evocações livres: uma temática de análise estrutural das representações sociais. In: MOREIRA, Antônia Silva Paredes. et. al. (Org.).Perspectivas teóricometodológicas em representações sociais. João Pessoa: Editora Universitária, 2005,p. 573-603.

OLIVEIRA, Denize Cristina de. et al. The building of a paradigm for nursing care lined in the human and health needs. Esc. Anna Nery, Rio de Janeiro, v. 15 , n. 4 , p. $838-844$, dez. 2011. Disponível em: $<$ http://www.scielo.br/pdf/ean/v15n4/a 25v15n4.pdf $>$. Acesso em: 15 abr. 2017.

SÁ, Celso Pereira de.et al. A memória histórica do regime militar ao longo de três gerações no Rio de Janeiro: sua estrutura representacional. Estud. psicol.(Campinas), Campinas, v. 26, n. 2, p. 159-171, jun. 2009. Disponível em:

<http://www.scielo.br/pdf/estpsi/v26n2 /04.pdf>. Acesso em: 16 fev. 2017.

SANTOS, Maria de Fátima S.;ALMEIDA, Leda Maria.

(Org.).Diálogos com a teoria da representação social.Recife. Ed. Universitária, 2005.

SARUBBI JUNIOR, Vicente.et al.Tecnologias computacionais para auxílio em pesquisa qualitativa - Sof- 
tware EVOC. São Paulo: Schoba, 2013.

SILVEIRA, E. A. A.; CARVALHO, A. M. P. Suporte relacionado ao cuidado em saúde ao doente com AIDS: o modelo de comboio e a enfermagem. Rev. esc. enferm. USP, São Paulo, v. 45, n. 3, p. 645-650, 2011. Disponível em:

<http://www.revistas.usp.br/reeusp/arti cle/viewFile/40747/44058>. Acesso em: 14 out. 2017.

SOUSA, C. S. O.; SILVA, A. L. O cuidado a pessoas com HIV/AIDS na perspectiva de profissionais de saúde. Rev. esc. enferm. USP, São Paulo, v. 47, n. 4, p. 907-914, 2013. Disponível em:

<http://www.scielo.br/pdf/reeusp/v47n 4/0080-6234-reeusp-47-4-0907.pdf > Acesso em: 10 abr. 2017.

SUTO,C. S. S. et al. O cuidado à Síndrome da Imunodeficiência Adquirida na contemporaneidade: compreendendo o olhar de profissionais. Rev Rene. v. 18, n. 6, p. 779-786, 2017. Disponível em:

$<$ http://periodicos.ufc.br/rene/article/vi ew/31090/71681>. Acesso em: 15 jan. 2018.

VERGÈS, P. L'évocation de l'argent: une méthodepourladéfinitiondunoyau central de lareprésentation. Bulletin de Psychologie, n. 45, 203-209, 1992.

Disponível em:

<http://psycnet.apa.org/record/1998-

01851-006>. Acesso em: 21 set. 2016.

WACHELKE, J.; WOLTER, R. Critérios de construção e relato da análise prototípica para representações sociais. Psic.: Teor. e Pesq., Brasília, v. 27, n. 4, p. 521-526, dez. 2011. Disponível em: $<\underline{\text { http://www.scielo.br/pdf/ptp/v27n4/1 }}$

7.pdf>. Acesso em: 21 set. 2016. 